\title{
Raus aus der «Black Box»
}

\author{
In der Psychiatrie wird in Zukunft ebenfalls nach Leistung abgegolten. Anders als in \\ der Akutsomatik gelten ab Anfang 2012 zwar (noch) keine diagnosebezogenen Fall- \\ pauschalen. Aber bis 2015 muss auch die Psychiatrie ein national einheitliches pau- \\ schales Abgeltungssystem einführen. Anfang April 2011 trafen sich Direktoren, Chef- \\ ärzte, und Pflegedirektoren zu einer gemeinsamen Lagebesprechung.
}

Irène Dietschi

Korrespondenz:

Dr. Graziella Giacometti Bickel Präsidentin Schweizerische

Vereinigung Psychiatrischer Chefärztinnen

und Chefärzte SVPC

Postgasse 17

CH-3000 Bern 8

Tel. 0313138849

welcome@

swissmentalhealthcare.ch
Was heisst eigentlich «Leistungsorientierung» für die Psychiatrie, und was bedeutet Multiprofessionalität in Zukunft? Dieser Frage widmete sich auf Einladung der Psychiatrischen Klinik Königsfelden in Brugg das erste Symposium von SwissMentalHealthcare (SMHC), der Vereinigung der Psychiatrischen Kliniken und Dienste der Schweiz (siehe Kasten). Ein spannendes Thema, denn zur Debatte stand nicht nur die Frage, wie man psychiatrische Leistungen adäquat berechnen soll, sondern auch, wie sich ein neues Tarifmodell auf die Zusammenarbeit der beteiligten Berufsgruppen auswirkt: Ändert sich das Rollenverständnis in den Klinikleitungen, von Ärzten, Pflegenden und Therapeuten an der Basis, wenn man leistungsbezogen arbeitet? Ändert sich die Beziehung zu den Patienten?

«Jeder sollte das tun, was er am besten kann, wichtig dabei ist die Durchlässigkeit», sagte einleitend SMHC-Präsidentin Ursula Fringer und Präsidentin der Klinikdirektoren, die in einer leistungsorientierten Finanzierung durchaus Chancen sieht. Grundlegend sei aber, dass künftige Leistungsaufträge nicht nur von den Politikern definiert würden, sondern gemeinsam in Diskussion mit der institutionellen Psychiatrie. Man müsse die Zusammenhänge erklären und aufzeigen, wie eine Institution funktioniere, sagte auch Harald Müller, der die Konferenz Pflegedirektorinnen und Pflegedirektoren Psychiatrischer Institutionen der Schweiz KPP präsidiert. «Für die Versicherungen zum Beispiel ist die Psychiatrie eine Black Box: Sie stecken Geld hinein, aber wissen nicht, was dabei herauskommt.»

\section{Leistungsbezogene Tagespauschalen}

Eine transparentere Finanzierung ist also gefragt. Aber wie? Auf der Basis von Diagnosen lassen sich die

\section{SwissMentalHealthcare}

SwissMentalHealthcare ist die gesamtschweizerische Vereinigung der Direktorinnen und Direktoren, der Chefärztinnen und Chefärzte sowie Pflegedirektorinnen und Pflegedirektoren der psychiatrischen Kliniken und Dienste. Sie versteht sich als Ansprechpartnerin der institutionellen Psychiatrie gegenüber allen Akteuren im Gesundheitswesen. Die für die Schweiz neuartige Allianz will sich für die institutionelle Psychiatrie im stationären wie im ambulanten Bereich sowie in Tagesklinken einsetzen. SwissMentalHealthcare unterstützt eine leistungsorientierte und -gerechte Finanzierung psychiatrischer Leistungen mittels gezielten Informationen und belegbaren Fakten.
Kosten in der Psychiatrie kaum prognostizieren: Darin waren sich die Referentinnen und Referenten an der Köngisfelder Tagung einig. Denn: Anders als in der Akutsomatik lässt sich der Genesungsprozess psychisch Kranker nicht vorhersagen. «Das Risiko von Rückfällen lässt sich ebenso wenig kalkulieren wie der Verlauf der Krankheit insgesamt», sagte Frau Dr. med. Graziella Giacometti Bickel, Präsidentin der Chefärztevereinigung (SVPC).

Eine Alternative zur fallbezogenen ist die tagesbezogene Pauschalierung. In Deutschland wird zurzeit ein Finanzierungssystem entwickelt, das als zentrales Element eine solche Tagespauschale für die stationäre und teilstationäre Behandlung aufweist, und zwar abhängig von den tatsächlich erbrachten Leistungen. Das System will dazu anreizen, Leistungen effektiv und gezielt einzusetzen («pay for performance»). Die Einführung ist für 2013 vorgesehen.

Aktuell werden in einem aufwendigen Kalkulationsprozess Leistungen beschrieben und deren Ressourcenverbrauch berechnet. An der SMHC-Tagung gab Georg Oppermann, Pflegedirektor am Bezirkskrankenhaus Augsburg und Mitglied im Deutschen Pflegerat, Einblick, wie das im Klinikalltag abläuft. So werden direkt erbrachte Leistungen (Therapie und Diagnostik) immer als Einheiten von 25 Minuten erfasst - nicht mehr und nicht weniger. Die restlichen Kosten, also auch die «blosse» Präsenz, werden in einem «Gewichtungsmodell» berechnet. Es wird zwischen Intensiv- und Regelbehandlung sowie zwischen wichtigen und Nebendiagnosen unterschieden. Weil für jeden Behandlungstag eine pauschalierte Abgeltung erhoben wird, führt dies beim einzelnen Patienten zu täglich wechselnden Tagespauschalen.

Das in der Erprobung stehende System macht den Beteiligten noch wenig Freude, wie Georg Oppermann festhielt. «Der bürokratische Aufwand ist massiv», sagte er. An seinem Spital seien für die Leistungserfassung eigens Dokumentalisten angestellt worden. Das Vergütungssystem erkenne aber nur, was im Rahmen der definierten Klassifikation erfasst und kodiert worden sei. «Das führt dazu, dass alle Beteiligten möglichst viele Therapie-Einheiten kodieren wollen und zum Teil sogar neu kreieren. Sie passen sich dem 25-Minuten-Takt an.» Ein «Up-Coding» sei nicht zu 
verhindern. Doch mehr Kodierung bedeute insgesamt nicht mehr Geld für die Psychiatrie.

Immerhin attestierte Oppermann dem System auch positive Aspekte: Es habe Ärzte, Pflegende und andere Berufsgruppen stärker zusammengeführt. Durch das gemeinsame Interesse ziehe man eher an einem Strang als bislang.

\section{Gleiche Massstäbe am CHUV}

Dass die genaue Dokumentation erbrachter Leistungen durchaus zur Qualität beitragen kann, verdeutlichte in seinem Referat Patrice Guex, Ärztlicher Direktor der Abteilung für Allgemeine Psychiatrie und des Departementes für Psychiatrie des Universitätsspitals Waadt (CHUV). Am CHUV wird die Psychiatrie nach genau denselben ökonomischen Ellen gemessen wie die übrigen Departemente. Monitoring der psychiatrischen Dienstleistungen und Qualitätsmanagement (gouvernance clinique) sind in der Waadt das A und O, denn: «Man muss sehr gut begründen, was man tut, um sein Budget zu rechtfertigen», erläuterte Patrice Guex.

Sein Departement arbeitet unter anderem mit dem aus Grossbritannien stammenden System HoNOS: einem Messinstrument, mit dem auf einer Skala von 1 bis 12 der Schweregrad eines psychiatrischen Problems erfasst und so die Bedürfnisse des Patienten eruiert werden. Die Mitarbeitenden schrecke es nicht $a b$, diese Bewertungen vorzunehmen, im Gegenteil: «Indem sie ihre Dienstleistungen dokumentieren, reflektieren sie ihre Tätigkeit bewusster», so Patrice Guex. Davon profitiere letztlich der Patient, der ja im Zentrum stehe.

Ein anderes Schlagwort an der SMHC-Tagung in Brugg war die «Multiprofessionalität». Die Organisatoren liessen sich dabei vom Gedanken leiten, dass ein neues Finanzierungssystem eine Chance sein könnte, überholte Strukturen aufzubrechen und zu neuen Formen der Zusammenarbeit zu finden. «Wer in den Kliniken macht was, wie und wo am besten? Diese Fragen wollen wir klären», lautete der Aufruf von Präsidentin Ursula Fringer.

\section{«Weihe des Arztes» unnötig?}

Dass die Antworten keineswegs eindeutig sind, zeigte das Gespräch zwischen Samuel Rom, Fachpsychologe für Psychotherapie FSP, und dem Psychiater Erich Seifritz, Klinikdirektor an der PUK Zürich. Samuel Rom, der als CEO die Klinik Schützen in Rheinfelden leitet, machte geltend, der medizinische Fokus in den Kliniken - und damit die Dominanz der Psychiater sei nicht mehr zeitgemäss. Rom machte sich insbesondere für seinen eigenen Berufsstand stark. «In den Kliniken arbeiten neben 500 Ärzten rund 350 Psychologen», sagte er. «Wir sind gutausgebildet, wir nehmen Untersuchungen und Therapien vor, bei uns steht der Patient ab dem ersten Tag einer langjährigen Ausbildung im Mittelpunkt, kurz: Wir leisten sehr gute Arbeit.» Das neue Psychologieberufegesetz attestiere den Psychologen, Probleme medizinisch zu lösen.
Es sei nicht akzeptabel, wenn Psychologen als «Lückenbüsser» für den Psychiatermangel herhalten müssten. «Wenn man unsere Expertise braucht, dann verlangen wir auch die entsprechenden Kompetenzen.» Die «höhere Weihe» des Arztes sei in einem multiprofessionellen Team nicht mehr relevant.

Erich Seifritz hielt dem entgegen, Samuel Rom unterschätze den medizinischen Anteil der psychiatrischen Ausbildung. «Unser Krankheitsverständnis stützt sich auf das bio-psycho-soziale Modell», erklärte Erich Seifritz. «Nach diesem Bild ist zwar die Psychotherapie ein wichtiger Bestandteil der Behandlung, aber sie deckt die biologischen und mitunter auch die sozialen Aspekte nicht ab.» An der PUK, wo stark interdisziplinär gearbeitet werde, hätten Psychologen an der unlängst eingerichteten Psychotherapie-Station manchmal die Fallführung - vorausgesetzt, «dass von medizinischer Seite gewährleistet ist, wo der Bedarf liegt». Ein möglicher Weg, Psychologen mit ärztlichen Kompetenzen auszustatten, sei ein Postgraduate-Studium über psychiatrisch relevante medizinische Inhalte, nach holländischem Vorbild. Andere Konstrukte gebe es in der Pflege, aber mit anderen Schnittstellen zur Medizin. «Ich habe nichts gegen interdisziplinäre Teams», sagte Seifritz, «aber ich wehre mich gegen Gleichmacherei.»

\section{Aufwertung der Pflege}

Egal, wie diese Auseinandersetzung ausgehen wird: Für den Patienten wird sich die psychiatrische Versorgung in Zukunft ohnehin verändern. Dies liegt unter anderem an einer demografiebedingten Zunahme der Fallzahlen, dem ein Mangel an Pflegenden und ein noch stärkerer Mangel an Ärzten gegenübersteht, wie Dirk Richter von der Berner Fachhochschule Gesundheit in seinem Referat über Pflegeperspektiven ausführte. Zu erwarten sei ein Übergang zu einer überwiegend ambulanten, integrierten Versorgung. Stärker als heute werde man künftig das Gewicht darauf legen, psychiatrischen Patienten dabei zu helfen, ihren sozialen Status und ihr soziales Netzwerk zu bewahren («Re-Inklusion»).

Die Ansätze, die Dirk Richter präsentierte, setzen auf das «Empowerment» von Patienten. Diese sollen in die Lage versetzt werden, sich selbst als «Handlungszentrum ihres Lebens» zu sehen, beispielsweise mittels Coaching oder einer Methode, die sich «Motivational Interviewing» nennt und deren Ziel die Selbstbefähigung durch Motivationssteigerung ist. Für solche Aufgaben seien Pflegende durchaus gerüstet, betonte der Dozent. Eine Studie an seiner Fachhochschule hatte ergeben, dass Pflegende die Qualität und den Nutzen ihrer Arbeit hauptsächlich in der Patientenorientierung sehen. «Die Psychiatrie der Zukunft muss sich in vielen Bereichen verändert ausrichten», resümierte Richter. Dabei könne die psychiatrische Pflege einen Teil der notwendigen und bis anhin nur unzureichend abgedeckten Aufgaben übernehmen und für sich neu definieren. Dadurch werde der Pflegeberuf durchaus aufgewertet. 